\title{
МНОГОВАРИАНТНОСТЬ СКОРОСТНОЙ МОДЕЛИ В ЗАДАЧЕ СТРУКТУРНЫХ ПОСТРОЕНИЙ ПО СЕЙСМИЧЕСКИМ И СКВАЖИННЫМ ДАННЫМ
}

\author{
А.П.СЫСОЕВ \\ Санкт-Петербургский горный университет, Санкт-Петербург, Россия
}

В работе обсуждаются особенности решения задачи структурных построений (прогноза глубин отражающих горизонтов) по системе сейсмических и скважинных данных. Сейсмические данные представлены значениями вертикального времени и скоростью суммирования, данные скважин - отметками глубины отражающих горизонтов. Вертикальное время и глубина отражающих горизонтов связаны уравнением средней скорости, но значение средней скорости не определяется в сейсмическом эксперименте, поэтому возникает задача подбора скоростной модели сложного природного объекта. Задача структурных построений решается подбором формальных конструкций, в которых находят корреляционные зависимости между параметрами глубинной модели среды и кинематическими параметрами волнового поля. Оптимальное решение задачи подбора модели определяется по минимальному расхождению прогнозных и фактических значений глубины по выборке скважин. На конкретном практическом примере представлены возможные варианты интерпретационной модели.

Обратная кинематическая задача в формате преобразования вертикального времени отраженных волн в глубину горизонтов решается в каждом производственном отчете по результатам сейсморазведочных работ и, вероятно, является наиболее распространенной задачей сейсморазведки. В силу многообразия объектов исследования и кажущейся очевидности решения эта тема слабо представлена в научной литературе.

Ключевые слова: метод отраженных волн; кинематическая интерпретация; скоростная модель; скорости суммирования

Как цитировать эту статью: Сысоев А.П. Многовариантность скоростной модели в задаче структурных построений по сейсмическим и скважинным данным // Записки Горного института. 2018. Т. 233. C. 459-470. DOI: 10.31897/PMI.2018.5.459

Введение. С момента становления метода отраженных волн (МOB) и по настоящее время структурные карты геологических границ являются необходимым и важнейшим результатом сейсмических исследований. Успехи решения задач структурной геологии определяются однозначным соответствием геологических объектов с событиями волнового поля, которые связаны между собой уравнениями геометрической сейсмики. Неизвестными в задаче структурных построений являются два типа параметров среды: скоростная модель и глубина точек отражения.

Пусть для нескольких отражающих горизонтов задана совокупность годографов отраженных волн. Для этой системы данных определим толстослоистую модель среды и способ решения прямой кинематической задачи. Задача структурной интерпретации состоит в оценке параметров структурно-скоростной модели среды на основе минимизации функционала, описывающего расхождение модельных и реальных годографов отраженных волн. В такой постановке в 80-е годы прошедшего столетия была реализована система кинематической интерпретации годографов КИНГ [5]. Задача подбора параметров глубинно-скоростной модели решалась на базе больших ЭВМ при полном отсутствии графических средств контроля решения и интерактива.

В принципе в этой же постановке, но уже на новом техническом уровне решается задача подбора параметров структурно-скоростной модели среды для глубинной миграции до суммирования по 3D-данным. Отметим, что задачи на поиск минимума всегда имеют решение, но для того, чтобы решение удовлетворяло априорным данным (отметкам глубин отражающих горизонтов ОГ, заданным в точках глубоких скважин), в пластовую модель среды вводят формальные параметры, учитывающие «анизотропию» скоростей. Но даже при значительном усложнении модели среды результаты глубинной миграции представляются не в глубинном, а во временно́м масштабе, поскольку при обработке сейсмических данных вопросы полного согласования сейсмических и скважинных данных пока не имеют удовлетворительного решения $[6,8]$. Это обстоятельство определяет актуальность анализа проблемы структурных построений на этапе интерпретации сейсмических данных.

В прикладных задачах структурной интерпретации рассматриваются два кинематических параметра годографа общей срединной точки: вертикальное время $t_{0}$ и скорость суммирования $v_{s}$. Кинематические параметры (временные разрезы амплитуд отраженных волн и разрезы скоростей суммирования) получают в процессе цифровой обработки сейсмических данных, а параметр $t_{0}$ 
Многовариантность скоростной модели в задаче структурных построений...

появляется как результат фазовой корреляции амплитуд отражений. Будем считать, что результат фазовой корреляции определяется однозначно по временным разрезам амплитуд, поэтому два кинематических параметра являются результатом обработки сейсмических данных.

Принимается, что вертикальное время $t_{0}$ описывает время прохождения луча по вертикали от линии приведения сейсмических наблюдений до отражающей границы и обратно без учета преломления лучей на границах раздела скоростной модели среды. Линия приведения может быть определена выше поверхности наблюдения - в этом случае в модель среды вводится фиктивный слой с заданной скоростью. Кроме того, в задачах компенсации латеральной неоднородности верхней части разреза выполняются процедуры замещения скоростей - изменение структуры и параметров скоростной модели среды и, соответственно, изменение вертикального времени отраженных волн.

В общем случае вертикальное время и эффективная скорость отраженных волн являются не результатом измерения параметров волнового поля в ходе выполнения натурного эксперимента, а представляют результат реконструкции поля на основе уравнений, описывающих распространение волн для определенной модели среды. Так, если переменный рельеф дневной поверхности компенсируется статическими поправками, то возникают искажения скорости суммирования, обусловленные ограничениями этого способа преобразования. Эффект искажения скорости отраженных волн возникает при компенсации статическими поправками погруженных неоднородностей разреза типа многолетнемерзлых пород. Следует также отметить широко известный факт искажения вертикального времени и эффективной скорости, обусловленный погрешностью определения низкочастотных статических поправок [7].

Даже без учета указанных факторов значения эффективной скорости соответствуют средней скорости только для однородной скоростной модели среды. Для реальных сред всегда имеет место вертикальный градиент скорости, поэтому различие между средними и эффективными скоростями нарастает с увеличением глубины ОГ. Для иллюстрации рассмотрим пример по материалам Томской области. Здесь в точках скважин по данным геоинформационной системы определены значения глубин опорного горизонта Б (кровля баженовской свиты) и в этих же точках по результатам сейсмических работ найдены вертикальное время и скорость суммирования $v_{s}$ отраженной волны. Полученные данные позволяют выполнить сравнительный анализ значений средней и эффективной скорости. Средние значения по выборке составили для средней скорости $2511 \mathrm{~m} / \mathrm{c}$, для скорости суммирования - $2721 \mathrm{~m} / \mathrm{c}$ (рис.1, $a$ ). Не существует аналитической формулы пересчета эффективной скорости в среднюю. Применение формул Урупова - Дикса для расчета интервальных скоростей с последующей оценкой значений средней скорости уменьшает различие между значениями скоростей, но не решает проблему полностью. Можно считать, что эффективная скорость (или скорость суммирования) является в задачах интерпретации атрибутом волнового поля, только корреляционно связанным с целевым параметром - средней скоростью до отражающих горизонтов.

Из анализа графиков скоростей следует, что различие между двумя последовательностями не описывается постоянным значением, т.е. мы не можем для приведения скорости суммирова-

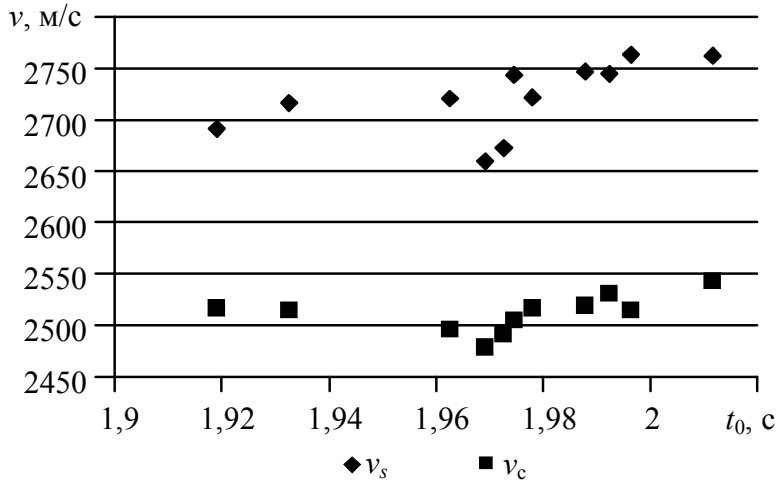

6

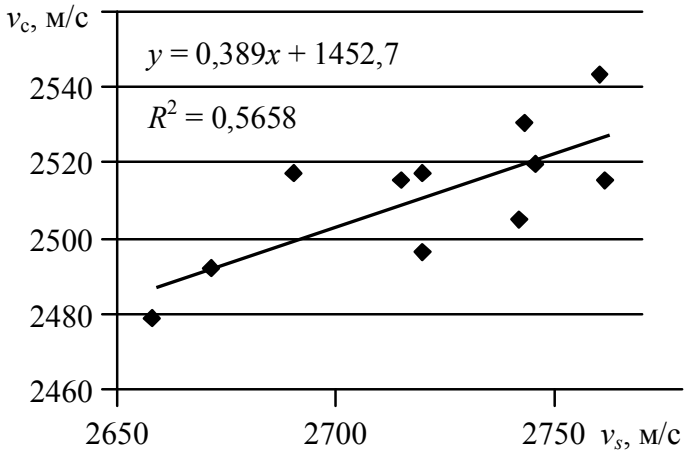

Рис. 1. Соотношение средних $v_{s}$ и эффективных $v_{\mathrm{c}}$ скоростей по горизонту Б 
ния к средней использовать уравнение вида $v_{\mathrm{c}}=v_{s}-a$. Также неудовлетворительно зависимость скоростей описывается уравнением вида $v_{\mathrm{c}}=a v_{s}$. Непротиворечивое описание распределения $v_{\mathrm{c}}\left(v_{s}\right)$ получено уравнением линейной регрессии (рис.1, б).

Определение задачи структурных построений. Следуя представлению С.В.Гольдина, задачу определения глубины отражающего горизонта можно представить следующим образом [2]. Пусть $D$ - область исследования в плоскости $(x, y)$. Будем считать, что в любой точке $M(x, y)$ определены параметры $t_{0}(M)$ и $v_{s}(M)$ - вертикальное время и эффективная скорость отраженной волны, являющиеся однозначными функциями переменной $M$. Глубина горизонта определяется уравнением

$$
h(M)=t_{0}(M) v_{\mathrm{c}}(M) / 2,
$$

где $v_{\mathrm{c}}(M)$ - средняя скорость до отражающей границы.

Для определения неизвестного параметра $v_{\mathrm{c}}(M)$ можно рассмотреть два варианта решения. Соотнесем значение средней скорости в точке $M$ со значением: а) вертикального времени $v_{\mathrm{c}}(M)=f\left(t_{0}(M)\right)$; б) эффективной скорости $v_{\mathrm{c}}(M)=f\left(v_{s}(M)\right)$.

В результате получим две постановки задачи структурных построений. В первом случае прогноз выполняется только по вертикальному времени отраженных волн, во втором исходными данными являются вертикальное время и эффективная скорость (скорость суммирования) отраженных волн.

Конкретная постановка задачи характеризуется определенной структурой исходных данных и моделью, связывающей неизвестные параметры с исходными данными. Исходные данные для рассматриваемой задачи включают скважинную и сейсмическую информацию: 1) скважинная информация - глубина и координаты пластопересечений целевых горизонтов; 2) сейсмическая информация - время слежения отражающих горизонтов и скорость суммирования ОГ.

Требуется выполнить: 1) прогноз поверхности, описывающей глубину целевого горизонта в пределах площади исследования; 2) оценку погрешности прогноза.

Особые условия: прогнозные значения глубины в точках пластопересечения должны точно совпадать с отбивками глубины в скважинах, если не доказана необходимость учета погрешности скважинных данных.

Графики сейсмокаротажа глубоких скважин также имеют непосредственное отношение к задаче структурных построений. Учет вертикальных годографов сейсмокаротажа представляет отдельный вариант задачи построения трехмерной скоростной модели среды, рассмотренный в работе [4].

Задача прогнозирования. Для оценки глубины сейсмического горизонта необходимо определить зависимость между переменными временного поля (вертикальное время $t_{0}$, скорость суммирования $v_{s}$ ) и прогнозируемыми параметрами (глубина $h$ или средняя скорость $v_{\mathrm{c}}$ ). Решение задачи прогноза разделим на четыре этапа.

1. Формирование обучающей выборки. Поскольку значения глубины заданы только в точках скважин, для получения обучающей выборки необходимо определить в этих точках значения сейсмических параметров. Дополнительно по значениям глубины и вертикального времени в точках скважин можно рассчитать значения средних скоростей: $v_{\mathrm{c}}=2 h / t_{0}$.

2. Подбор модели. Для решения задачи необходимо определить, как минимум, одну скоростную модель среды, связывающую кинематические параметры отраженных волн со средней скоростью либо непосредственно глубиной горизонтов. Оценка параметров модели выполняется минимизацией значения функции, описывающей отклонение прогнозных и фактических глубин в точках скважин. Также допустима постановка задачи подбора параметров модели, в которой минимизируется отклонение прогнозного и наблюденного времени.

3. Расчет параметров глубинно-скоростной модели среды в области определения сейсмических данных.

4. Согласование прогнозных и фактических значений глубины в точках скважин (разброс невязок).

Обоснование линейной зависимости глубины от вертикального времени ОГ. Уравнение линейной регрессии наиболее часто применяется при анализе зависимости «глубина - время» 
Многовариантность скоростной модели в задаче структурных построений...

отражающего горизонта. Покажем, что эта формальная конструкция физически содержательна для описания определенного типа структурной модели среды.

Пусть в $K$ скважинах известны глубина $h$ и значение вертикального времени $t_{0}$. Определим зависимость глубины и вертикального времени уравнением линейной регрессии

$$
h=a t_{0}+b .
$$

Отметим, что для вертикального годографа сейсмического каротажа (СК) зависимость $h(t)$ определяется непрерывной возрастающей функцией, которая допускает кусочно-линейную аппроксимацию, соответствующую пластовой модели среды. Следовательно, для данных СК представление зависимости «время - глубина» уравнением линейной регрессии в пределах однородного пласта обоснованно.

Уравнение (1) представляет однозначную зависимость глубины от времени отражения. Следуя [1] продифференцируем функцию по $t_{0}$ :

$$
\frac{d h}{d t_{0}}=a=\text { const. }
$$

Параметр $a$ характеризует скорость изменения глубины границы от вертикального времени. Для краткости этот параметр будем называть «градиентом» линейной функции (1). Для слоистой модели среды с постоянными скоростями слоев $\left(h(x)=\sum h_{i}(x) ; t_{0}(x)=2 \sum h_{i}(x) / v_{i}\right)$ градиент определяется выражением

$$
\frac{d h}{d t_{0}}=\frac{d h}{d x} \frac{d x}{d t_{0}}=\frac{d h}{d x} / \frac{d t_{0}}{d x}=\sum_{i} \frac{d h_{i}}{d x} / \sum_{i} \frac{2 d h_{i}}{v_{i} d x} .
$$

Нетрудно показать, что выражение (3) имеет постоянное значение, если мощности слоев определяются линейно зависимыми функциями вида

$$
h_{i}(x)=d_{i} f(x)+c_{i}
$$

с произвольными коэффициентами $d_{i}, c_{i}$. Тогда

$$
\frac{d h}{d t_{0}}=\sum_{i} d_{i} / \sum_{i} \frac{2 d_{i}}{v_{i}}=a=\text { const } .
$$

Рассмотрим частный случай - двухслойную модель, где мощности двух слоев с постоянной скоростью описываются произвольными функциями координаты профиля:

$$
h_{1}(x)=d_{1} f(x)+c_{1} ; \quad h_{2}(x)=d_{2} f(x)+c_{2} .
$$

Соответственно, глубина и вертикальное время для нижней границы определяются выражениями:

$$
\begin{gathered}
h(x)=\left(d_{1}+d_{2}\right) f(x)+\left(c_{1}+c_{2}\right) \\
t_{0}(x)=2\left(\left(d_{1} / v_{1}+d_{2} / v_{2}\right) f(x)+\left(c_{1} / v_{1}+c_{2} / v_{2}\right)\right) .
\end{gathered}
$$

Уравнение (4) приводится к виду

$$
\frac{d h}{d t_{0}}=0,5\left(d_{1}+d_{2}\right) /\left(d_{1} / v_{1}+d_{2} / v_{2}\right)=a=\text { const. }
$$

Из полученного выражения (5) следует, что зависимость $h\left(t_{0}\right)$ может быть возрастающей $(a>0)$, убывающей $(a<0)$ и постоянной $(a=0)$ функцией вертикального времени.

Выразим значение градиента $a$ как функцию коэффициента $k$, характеризующего отношение мощностей слоев глубинной модели среды:

$$
k=d_{2} / d_{1}, \Rightarrow a=\frac{1+k}{2\left(1 / v_{1}+k / v_{2}\right)} .
$$


Многовариантность скоростной модели в задаче структурных построений...

Положительное значение коэффициента $k$ соответствует прямой линейной зависимости между мощностями слоев, отрицательное - обратной. Прямая зависимость отражает унаследованное развитие толщ, обратная зависимость не имеет простого геологического истолкования, но в принципе интересна для формального анализа. График зависимости $a(k)$ для двухслойной среды с пластовыми скоростями $v_{1}=2000 \mathrm{M} / \mathrm{c}, \quad v_{2}=3000 \mathrm{M} / \mathrm{c}$ представлен на рис. 2 .

Отметим следующие особенности:

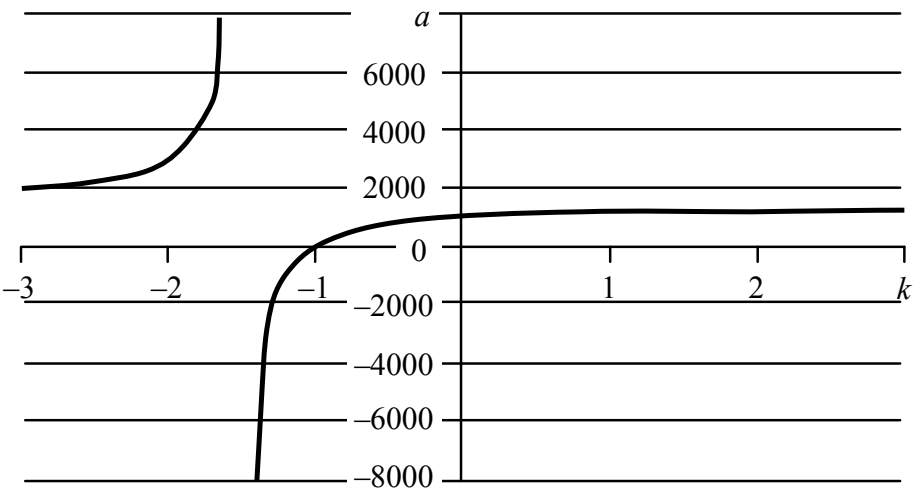

Рис.2. График зависимости градиента $a$ от отношения $k=d_{2} / d_{1}$ параметров глубинной модели

1. В точке $k=-1,5$ график функции имеет разрыв $\pm \infty$, здесь знаменатель выражения (6) равен нулю:

$$
1 / v_{1}+k / v_{2}=0, \Rightarrow k=-v_{2} / v_{1}, \Rightarrow k=d_{2} / d_{1}=-v_{2} / v_{1}, \Rightarrow d_{2}=-d_{1} v_{2} / v_{1} .
$$

Подставив выражение для $d_{2}$ в уравнения (5), определим, что точка разрыва соответствует переменной глубине ОГ при постоянном значении вертикального времени отражающего горизонта (рис.3):

$$
\begin{gathered}
h(x)=d_{1}\left(1+d_{2} v_{2} / v_{1}\right) f(x)+\left(c_{1}+c_{2}\right) ; \\
t_{0}(x)=2\left(c_{1} / v_{1}+c_{2} / v_{2}\right)=\text { const. }
\end{gathered}
$$

Резкое изменение графика в окрестности точки разрыва $k=-v_{2} / v_{1}$ определяется сколько угодно большим изменением глубины при малом изменении вертикального времени.

2. Градиент $a$ равен нулю в точке $k=-1$, что соответствует отношению $d_{2}=-d_{1}$. Из уравнений (5) получим, что значение $a=0$ соответствует постоянной глубине при переменном значении вертикального времени (рис.4).

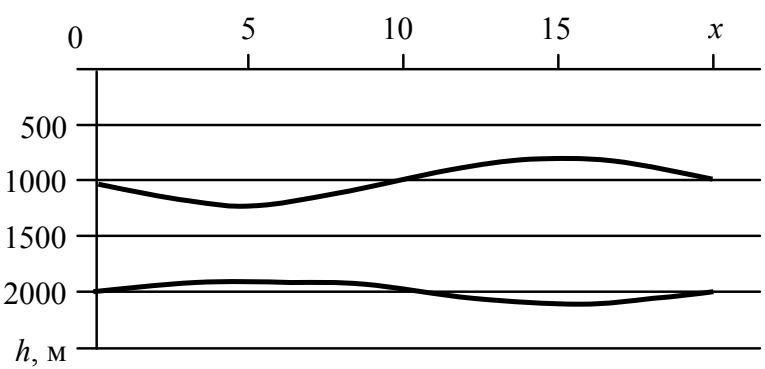

6

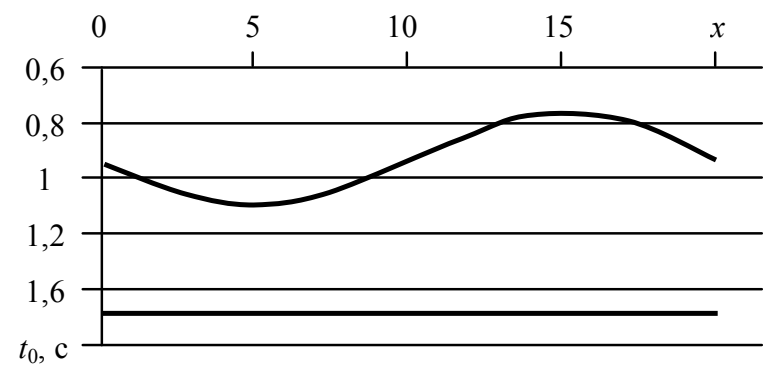

Рис.3. Глубинная модель при $k=-v_{2} / v_{1}(a)$ и графики вертикального времени ОГ (б)
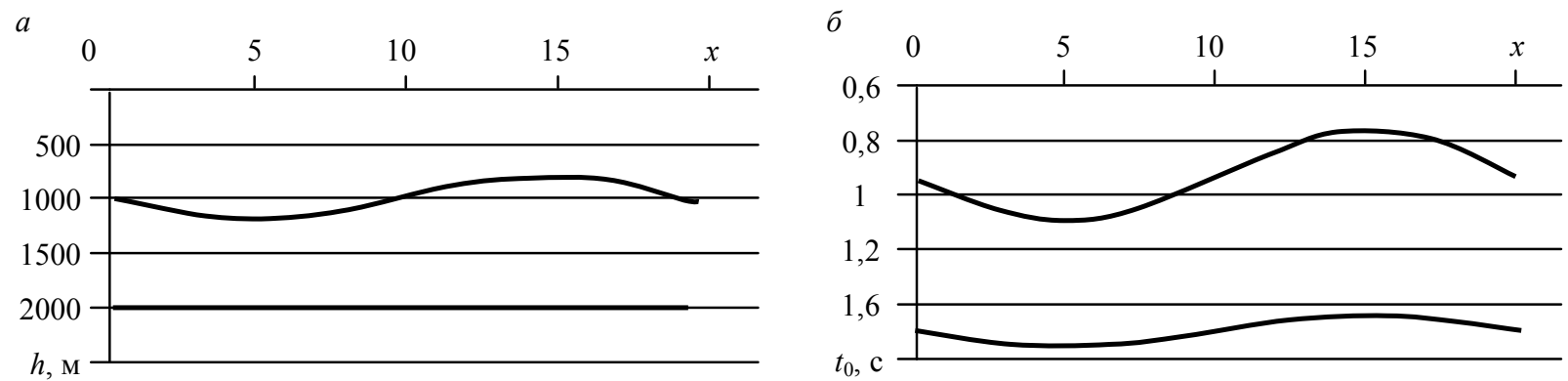

Рис.4. Глубинная модель при $k=-1$ (a) и графики вертикального времени ОГ (б) 
Многовариантность скоростной модели в задаче структурных построений...

3. В диапазоне $-1,5<k<-1$ градиент $a$ принимает отрицательное значение, что соответствует уменьшению глубины с увеличением вертикального времени ОГ (рис.5).

4. Точка пересечения оси ординат $(k=0)$ соответствует постоянной мощности второго слоя. Значение градиента $a=v_{1} / 2=1000 \mathrm{~m} / \mathrm{c}$ определяется скоростью первого слоя (рис.6).

5. Асимптотика $a(k \rightarrow \pm \infty) \rightarrow v_{2} / 2=1500 \mathrm{~m} / \mathrm{c}$ соответствует модели, в которой изменение мощности первого слоя мало, сравнительно с вариацией мощности второго (рис.7). В пределе этому варианту модели соответствует постоянная мощность первого слоя.

В заключение этого раздела рассмотрим модель среды со слабо выраженной зависимостью мощности слоев. Линейная зависимость $h\left(t_{0}\right)$ определяется, но проявляются отклонения значений глубины от линии регрессии (рис.8), интерпретируемые как ошибки прогноза.

По результатам модельных экспериментов можно сделать вывод, что уравнению линейной зависимости между глубиной и вертикальным временем отражения соответствует в общем случае многослойная модель среды с линейной связью между мощностями слоев. Признаком этого

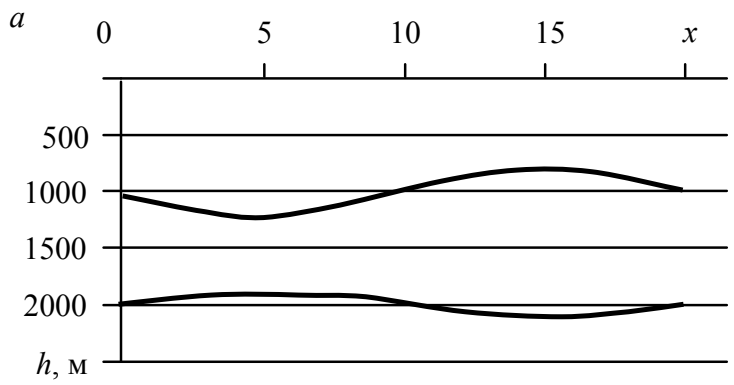

$\sigma$

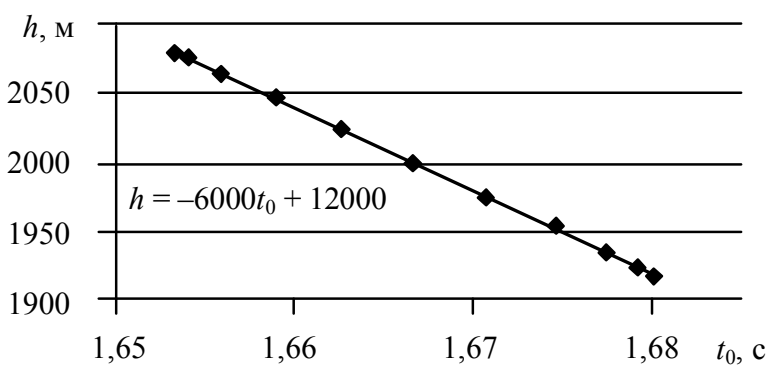

Рис.5. Глубинная модель при $k=-1,4$ (a) и график зависимости $h\left(t_{0}\right)($ б)
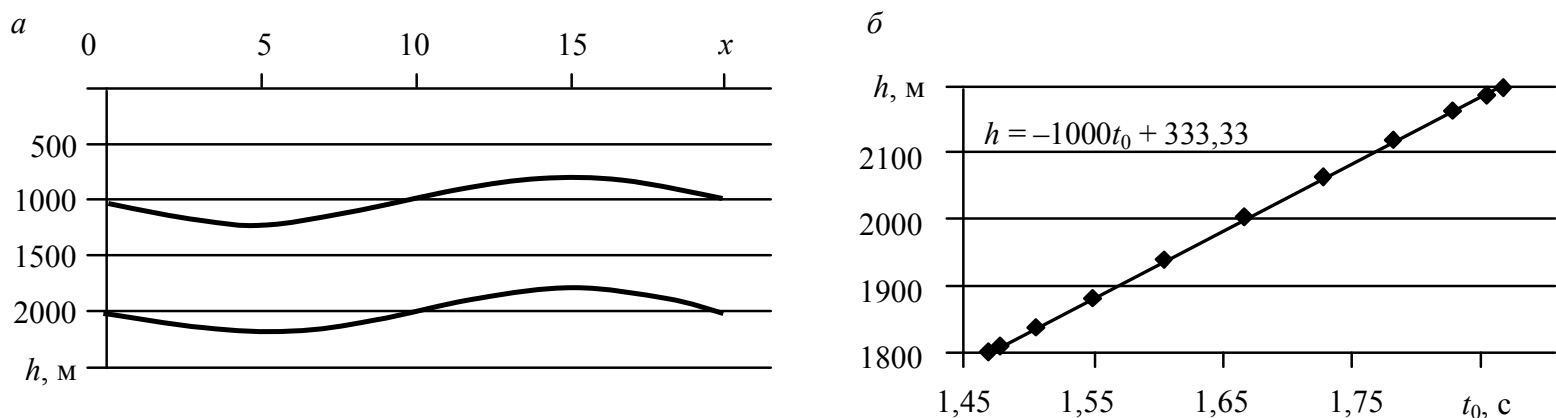

Рис.6. Глубинная модель при $k=0$ (a) и график зависимости $h\left(t_{0}\right)($ б)
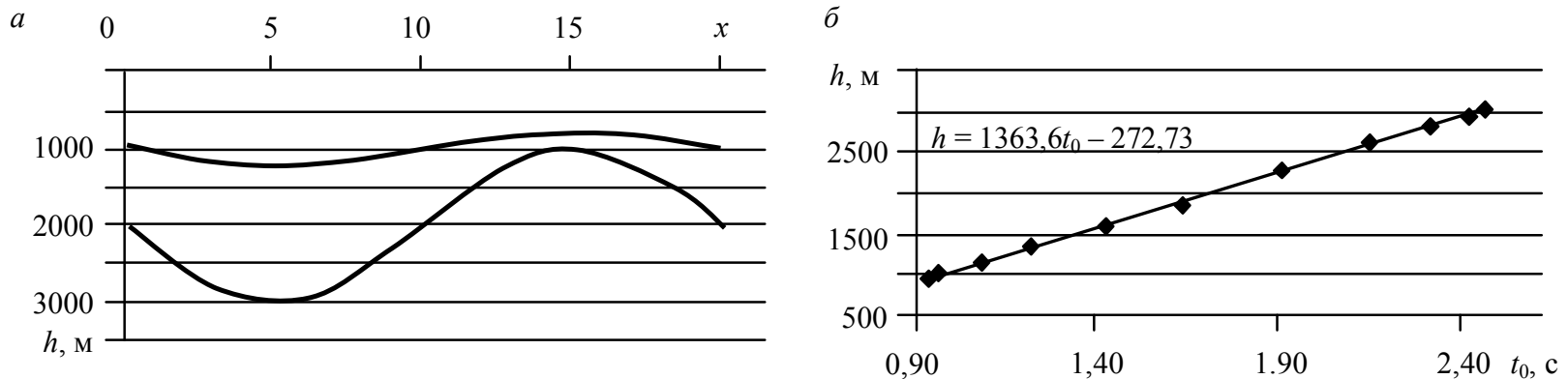

Рис.7. Глубинная модель при $k=4$ (a) и график зависимости $h\left(t_{0}\right)($ б) 
Многовариантность скоростной модели в задаче структурных построений...

типа моделей может служить линейная зависимость между интервальными мощностями или значениями времени отражающих горизонтов.

Подбор интерпретационной модели. Варианты подбора скоростной модели проиллюстрируем на примере обучающей выборки по одной из площадей Томской области. Здесь в 11 скважинах глубокого бурения определены значения вертикального времени и отбивок глубин горизонтов с условными индексами Г, Б. Значения времени ОГ, упорядоченные по возрастанию второй переменной, представлены на рис.9. Для целевого горизонта Б также определены значения скоростей суммирования, представленные на рис.2.

На первом этапе анализа предположим, что в $K$ точках скважин известен прогнозируемый параметр $h_{k}$, значение переменной $t_{0 k}$ и расчетное значение средней скорости $v_{k}$ для горизонта Б. Даже для этой простой системы данных можно предложить несколько вариантов глубинноскоростной модели среды.

1. Распределение средней скорости $v(\mathbf{x})$ по площади исследования можно получить интерполяцией значений средней скорости, рассчитанных в точках скважин.

2. Значение скорости определяется как функция вертикального времени отражения: $v(\mathbf{x})=f\left(t_{0}(\mathbf{x})\right)$.

3. Глубина горизонта определяется как функция вертикального времени отражения: $h(\mathbf{x})=f\left(t_{0}(\mathbf{x})\right)$.

4. Вертикальное время отражения описывается как функция глубины горизонта: $t_{0}(\mathbf{x})=f(h(\mathbf{x}))$.
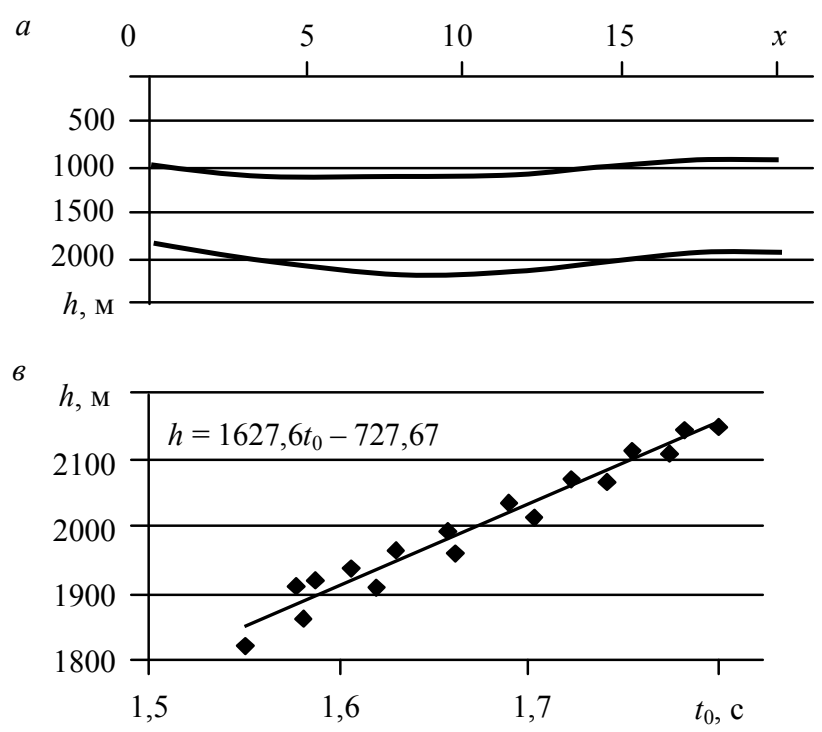

Номер скважины

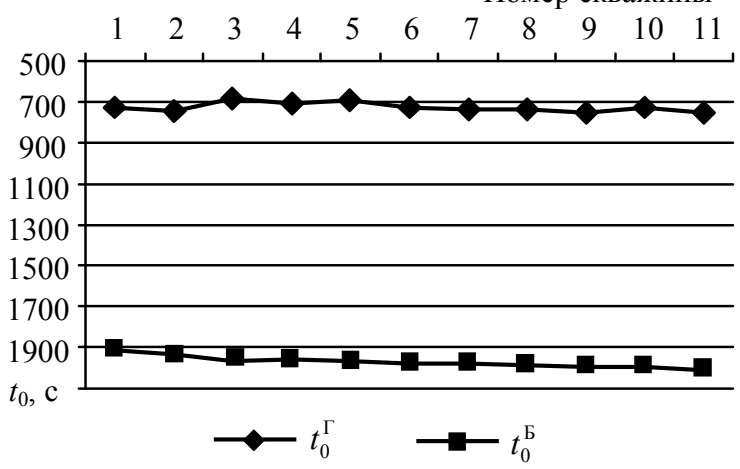

Рис.9. Значения вертикального времени в точках скважин
6

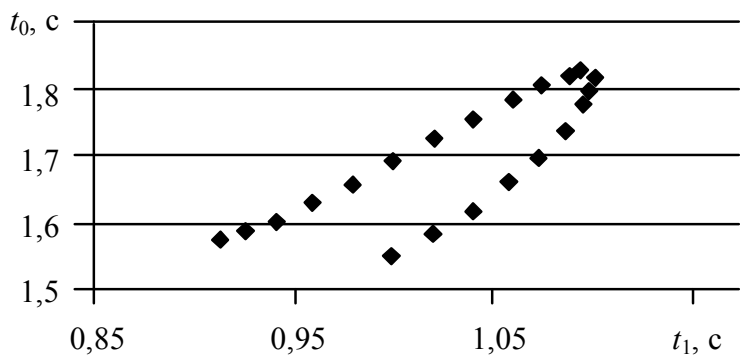

Рис.8. Глубинная модель с нелинейной зависимостью мощности слоев $(a)$, график зависимости $t_{0}\left(t_{1}\right)(\sigma)$; распределение $h\left(t_{0}\right)(8)$

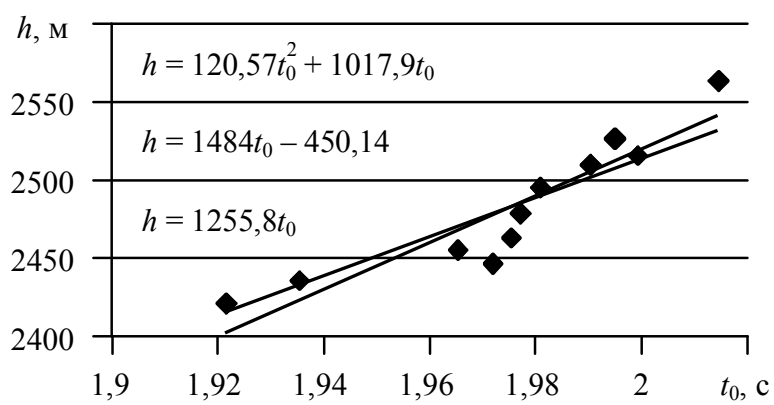

Рис.10. Распределение $h\left(t_{0}\right)$ по выборке скважин 
Многовариантность скоростной модели в задаче структурных построений...

Модель средней скорости $v(\mathbf{x})$. Самое простое решение задачи сводится к интерполяции средней скорости по множеству значений $v_{k}$. Модель средней скорости, соответствующая этому решению, описывается выражением, включающим постоянную $v_{0}$ и переменную составляющие:

$$
v_{k}=v_{0}+e_{v k} .
$$

Оценка параметра $v_{0}$ определяется из условия минимума функционала, описывающего расхождение фактических и прогнозных глубин в точках скважин:

$$
\Theta\left(v_{0}\right)=\sum\left(h_{k}-v_{0} t_{0 k} / 2\right)^{2} \rightarrow \min , \Rightarrow \widehat{v}_{0}=\sum_{k=1}^{K} h_{k} t_{0 k} / \sum_{k=1}^{K}\left(t_{0 k}^{2} / 2\right) .
$$

Используя оценку скорости $\widehat{v}_{0}$, определим для каждой скважины расхождение $e_{h}$ прогнозной и фактической глубины и значение стандартной погрешности прогноза $\sigma_{h}$ :

$$
e_{h k}=h_{k}-\widehat{v}_{0} t_{0 k} / 2 ; \quad \sigma_{h}^{2}=\sum_{k=1}^{K} e_{h k}^{2} /(K-1) .
$$

На рис.10 модель средней скорости для горизонта Б описывается уравнением $h=1255,8 t_{0}$. Стандартная погрешность прогноза по выборке $\sigma_{h}=17,9$ м.

Модель линейной регрессии $v\left(t_{0}\right)$. Уравнение линейной зависимости средней скорости от времени отражения преобразуем к зависимости глубины от времени отражения:

$$
v=a t_{0}+b, \Rightarrow v t_{0} / 2=\left(a t_{0}+b\right) t_{0} / 2, \Rightarrow h\left(t_{0}\right)=d t_{0}^{2}+c t_{0} .
$$

В ней глубина выражается параболическим уравнением с нулевым постоянным членом (рис.10). Стандартная погрешность прогноза глубины по выборке $\sigma_{h}=16,7$ м.

Модель линейной регрессии $h\left(t_{0}\right)$. Результат аппроксимации зависимости $h\left(t_{0}\right)$ уравнением линейной регрессии

$$
h=a t_{0}-b+e_{h}
$$

представлен на рис.10. На интервале изменения переменной $t_{0}$ линия регрессии практически накладывается на уравнение параболы (7). Стандартная погрешность прогноза по выборке $\sigma_{h}=16,8 \mathrm{M}$.

Peгрессия $t_{0}(h)$. В рассмотренном выше уравнении линейной регрессии (8) случайная составляющая $e_{h}$ определяется как погрешность измерения глубины горизонтов $h$ в точках скважин, а объясняющая переменная $t_{0}$ считается заданной точно. Предположение, что глубина скважин известна точно, а время отражений содержит погрешности, более соответствует реальной ситуации. В работе [3] эта ситуация описывается как «ошибки объясняющих переменных», которые приводят к смещению оценок параметров уравнения линейной регрессии. Если $\sigma_{t}-$ среднеквадратичная ошибка переменной $t_{0}$, то смещение коэффициента $a$ оценивается выражением $a \sigma_{t}^{2} /\left(\sigma_{t}^{2}+\sigma_{h}^{2}\right)$.

Указанное противоречие модели можно преодолеть, если уравнение линейной регрессии использовать для описания вертикального времени как функции глубины горизонта:

$$
t_{0}=a h+b+e_{t} .
$$

После оценки коэффициентов уравнение регрессии $t_{0}(h)$ приведем к представлению

$$
h\left(t_{0}\right)=t_{0} / \hat{a}-\hat{b} / \hat{a} .
$$

Коэффициенты регрессии подбираются из условия минимума расхождения прогнозных и фактических значений времени отражения, поэтому погрешность прогноза глубины по уравнению (9) возрастает до 18,2 м.

Таким образом, по одной выборке данных получено две линейные зависимости глубины горизонта от вертикального времени отраженной волны. Предположим, что вертикальное время отражения в пределах площади исследования изменяется в диапазоне 1,9-2,1 с и выполним расчет глубины горизонта с использованием уравнения прямой $h\left(t_{0}\right)$ и обратной $t_{0}(h)$ зависимостей 
Многовариантность скоростной модели в задаче структурных построений...

(рис.11). Расхождение между решениями на заданном интервале изменения времени отражения $\left(0,2\right.$ c) составляет от -18 до 33 м. Зависимость $t_{0}(h)$ определяет решение с более резким изменением гипсометрии отражающей границы.

Двухслойная модель среды. Введем в условие задачи значения вертикального времени и отбивки глубины отражающего горизонта Г. Естественно ожидать, что параметризация глубинной модели двумя слоями позволит уменьшить погрешность прогноза целевого горизонта Б.

Ранее показано, что уравнение линейной регрессии корректно описывает зависимость глубины от вертикального времени, если существует линейная зависимость между значениями глубины (или вертикального времени границ). На рис.12 представлен график совместного распределения $t_{0}^{\mathrm{Б}}\left(t_{0}^{\Gamma}\right)$ вертикальных времен двух горизонтов. Линейная зависимость между значениями времени двух горизонтов отсутствует, что является причиной значительной погрешности прогноза глубины по уравнению линейной регрессии.

Если временные мощности двух интервалов разреза изменяются независимо, то логично рассмотреть модель с независимой оценкой мощности каждого интервала. На рис.13 представлены два графика, характеризующие распределение $h\left(t_{0}\right)$ для горизонта $\Gamma$ и зависимости $d_{h}\left(d_{t}\right)$ мощности интервала Б-Г от интервального времени.

Для горизонта $\Gamma$ (рис.13,a) зависимость $h\left(t_{0}\right)$ удовлетворительно описывается уравнением линейной регрессии, значение стандартной погрешности $\sigma_{h}=4,2$ м. Для интервала Б-Г линейная зависимость определяется низким значением коэффициента детерминации $R^{2}=0,49$ и погрешность прогноза мощности интервала $\sigma_{h}=24$ м. Таким образом, усложнение модели, выполненное разделением среды на два интервала, приводит к увеличению погрешности прогноза глубины горизонта Б, сравнительно с линейной зависимостью от одной переменной.

В рассматриваемой задаче заданы всего два горизонта, поэтому перебором объясняющих переменных и параметров модели несложно установить, что для оценки мощности интервала Б-Г

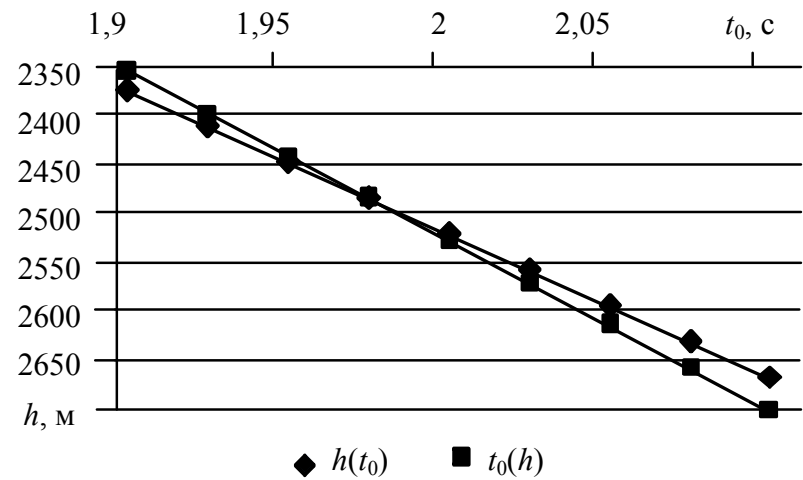

Рис.11. Расхождение оценки глубины ОГ для моделей регрессии $h\left(t_{0}\right)$ и $t_{0}(h)$

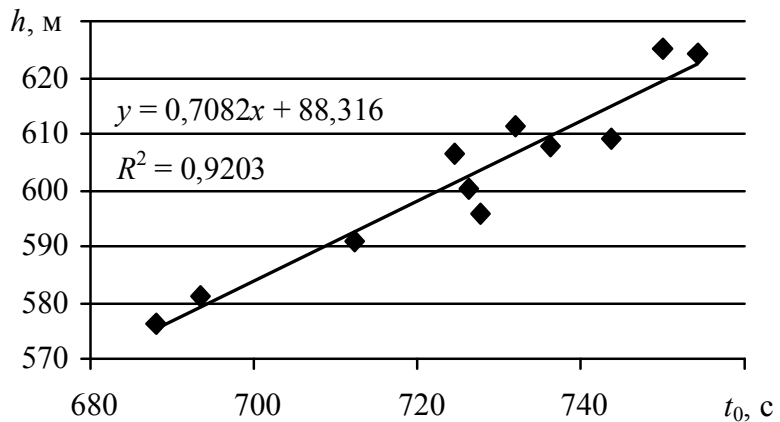

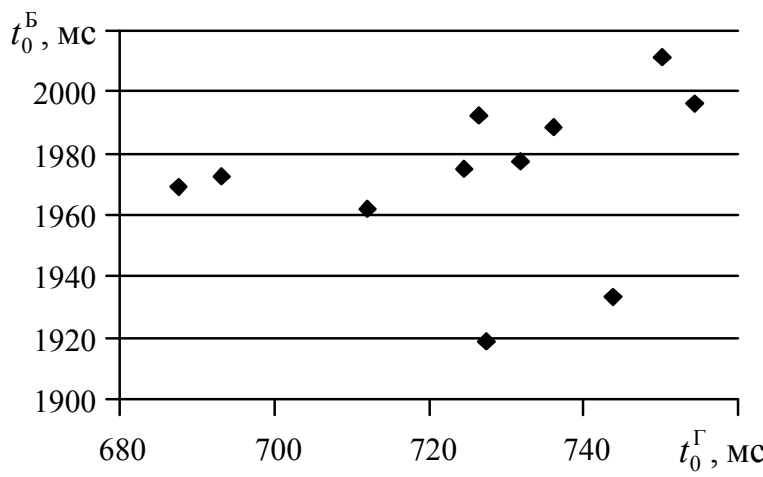

Рис.12. Распределение $t_{0}^{\mathrm{Б}}\left(t_{0}^{\Gamma}\right)$ в точках скважин

$\sigma$

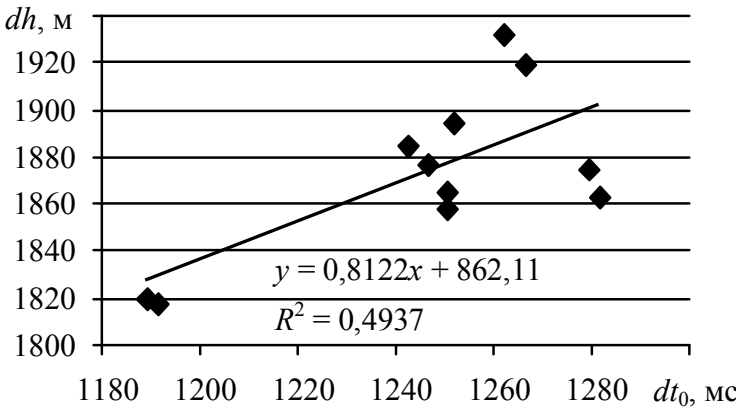

Рис.13. Анализ зависимостей для отражающего горизонта Г( $a)$ и интервала Б-Г(б) 


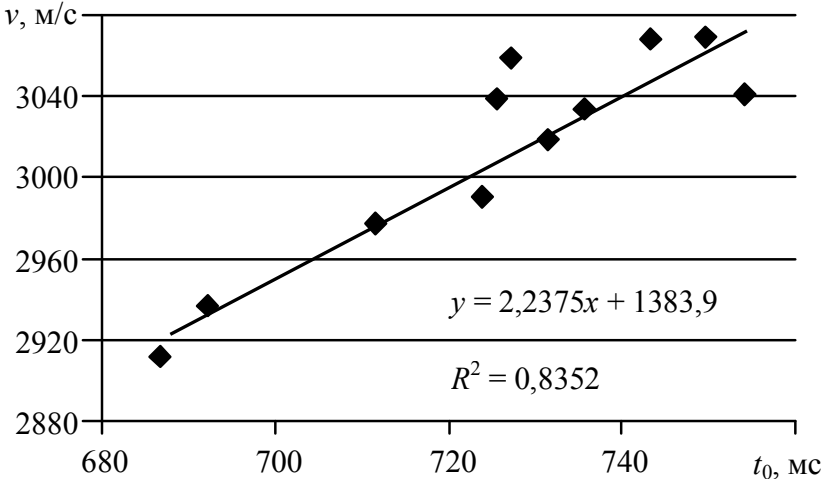

Рис.14. Зависимость средней скорости интервала Б-Г от $t_{0}$ горизонта $\Gamma$

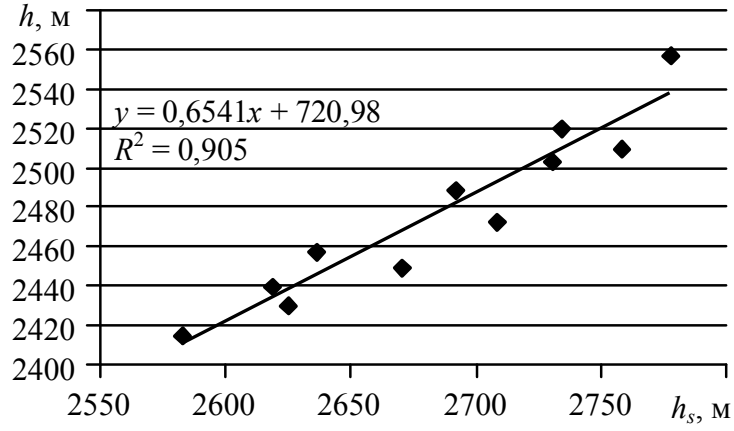

Рис.15. Зависимости фактической глубины горизонта от «эффективной»

определяется практически важный вариант модели в виде линейной зависимость средней скорости интервала Б-Г от вертикального времени гор. Г - кровли анализируемого интервала (рис.14)

$$
v^{\mathrm{L}-\Gamma}=a_{0}+a_{1} t_{0}^{\Gamma} \Rightarrow \hat{d}_{h}{ }^{\mathrm{L}-\Gamma}=\hat{v}^{\mathrm{L}-\Gamma}\left(t_{0}^{\mathrm{L}}-t_{0}^{\Gamma}\right) / 2 .
$$

В этом варианте решения мощность первого слоя оцениваем уравнением линейной регрессии, а скоростную модель нижнего интервала описываем уравнением средней скорости $v^{\text {Б-Г }}\left(t_{0}^{\Gamma}\right)$. Стандартная погрешность прогноза глубины горизонта Б для двухслойной модели среды

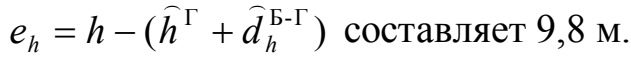

Модель эффективной скорости. Рассмотрим задачу, в которой сейсмические данные представлены функциями вертикального времени и скорости суммирования отраженной волны: $t_{0}(\mathbf{x}), v_{s}(\mathbf{x})$. Кроме того, в точках скважин рассчитаны значения средней скорости $v_{\mathrm{c}}$.

Для пересчета скорости суммирования к средней скорости используем формальное описание уравнением линейной регрессии $v_{c}=a v_{s}+b+e_{v}$, где $e_{v}$ - случайная составляющая. График распределения $v_{\mathrm{c}}\left(v_{s}\right)$ приведен на рис. $1, \sigma$. Глубина горизонта определится по формуле

$$
\hat{h}(\mathbf{x})=\widehat{v}_{c}(\mathbf{x}) t_{0}(\mathbf{x}) / 2=\left(\hat{a} v_{s}(\mathbf{x})+\widehat{b}\right) t_{0}(\mathbf{x}) / 2 .
$$

Значение стандартной погрешности прогноза глубины для этой модели $\sigma_{h}=11,69$ м.

Модель эффективной глубины. В предыдущей задаче выполнялась подгонка скорости суммирования к средней скорости. Между тем конечная цель решения состоит в подборе скоростной модели, которая минимизирует расхождение глубин в точках скважин.

В точках сейсмических наблюдений известны значения вертикального времени и скорости суммирования (эффективные скорости). Это позволяет рассчитать функцию, которую назовем «эффективной глубиной» горизонта: $h_{s}=v_{s} t_{0} / 2$. Значения $h_{s}$ смещены относительно истинных глубин, поскольку скорости суммирования смещены относительно средних скоростей. Но значения $h_{s}$ можно использовать как объясняющие переменные уравнения регрессии для прогноза глубины горизонта (рис.15):

$$
h=d_{1} h_{s}+d_{2}=d_{1}\left(v_{s} t_{0} / 2\right)+d_{2}+e_{h} .
$$

Зависимость $h\left(h_{s}\right)$ характеризуется более высоким коэффициентом детерминации, чем при оценке средней скорости в предыдущем примере, но значение погрешности прогноза глубины $\sigma_{h}=13,42$ м больше, чем для модели эффективной скорости.

Как учесть случайный характер результата. Принимаем во внимание, что коэффициенты уравнения регрессии и ошибки прогноза являются случайными величинами, т.е. минимальная погрешность, определяемая по ограниченной выборке, не является гарантией лучшего решения. Можно рекомендовать выполнить расчет всех вариантов и оценить расхождение между картами глубин. Возможно, что расхождение будет незначительным, тогда проблема выбора модели отпадает. При значительной разнице допустимо получить средневзвешенное значение нескольких вариантов решения. 
Многовариантность скоростной модели в задаче структурных построений...

Если принять условие независимости ошибки прогноза для определенных типов модели, то можно предложить усреднение карт с учетом погрешности прогноза каждой реализации. Пусть, например, получено два заслуживающих внимания результата: модель эффективной скорости (10) и двухслойной модели с оценкой интервальной скорости (11). Оценки погрешности решения, определяемые по выборке из 11 скважин, составляют соответственно 11,7 и 9,8 м.

На множестве точек $\mathbf{x}$ определены два варианта решения $h_{1}(\mathbf{x}), h_{2}(\mathbf{x})$, для которых определены оценки дисперсии ошибки прогноза: $D_{1}, D_{2}$. Значение искомой функции в произвольной точке $\mathbf{x}$ определим как взвешенную сумму исходных данных:

$$
h(\mathbf{x})=w_{1} h_{1}(\mathbf{x})+w_{2} h_{2}(\mathbf{x}),
$$

где $w_{1}+w_{2}=1$.

Дисперсия суммы двух независимых величин определяется выражением

$$
D_{e}=w_{1}^{2} D_{1}+w_{2}^{2} D_{2} .
$$

Задача определения весов $w_{1}, w_{2}$, обеспечивающих минимум дисперсии с учетом дополнительного условия, определяется минимизацией функционала:

$$
D\left(w_{1}, w_{2}, \lambda\right)=w_{1}^{2} D_{1}+w_{2}^{2} D_{2}+\lambda\left(w_{1}+w_{2}-1\right),
$$

где $\lambda$ - неопределенный множитель Лагранжа [1].

Точка минимума функционала определяется условием равенства нулю частных производных:

$$
\frac{\partial}{\partial w_{1}} D\left(w_{1}, w_{2}, \lambda\right)=0 ; \quad \frac{\partial}{\partial w_{2}} D\left(w_{1}, w_{2}, \lambda\right)=0 ; \quad \frac{\partial}{\partial \lambda} D\left(w_{1}, w_{2}, \lambda\right)=0 .
$$

Несложно определить, что весовые множители определяются выражениями

$$
w_{1}=D_{2} /\left(D_{1}+D_{2}\right) ; \quad w_{2}=D_{1} /\left(D_{1}+D_{2}\right) .
$$

Оценка стандартной погрешности прогноза глубины целевого горизонта, рассчитанной по формуле (11), $\sigma_{h}=7,5 \mathrm{~m}$.

Заключение. Оценка глубины сейсмических горизонтов представляет стандартную задачу прогноза, где в качестве объясняющих переменных используются кинематические параметры волнового поля: вертикальное время и скорость суммирования отраженных волн. Отсутствие функциональной зависимости между прогнозируемыми параметрами среды и параметрами волнового поля определяет многовариантность описания зависимости глубины горизонта от кинематических параметров волнового поля. Варианты модели определяются структурой модели и исходных данных. Критерием оптимального выбора варианта модели является минимальное расхождение прогнозных и фактических глубин, заданных в точках скважин. Как правило, структурные построения выполняются по серии отражений, что определяет возможность решения задачи в рамках однослойной или многослойной модели среды.

Стандартным вариантом однослойной модели является уравнение линейной зависимости глубины от вертикального времени. Показано, что это уравнение соответствует многослойной модели среды при условии линейной зависимости мощности слоев. Отмечено, что значения параметров модели линейной регрессии согласуются с параметрами толстослоистой модели среды, определяемыми по данным сейсмокаротажа, только в предельных вариантах скоростной модели.

Ограничения на структуру модели снимаются при использовании эффективной скорости отраженных волн, но этот параметр редко применяется при решении практических задач, поскольку оценки скорости суммирования неустойчивы к погрешности параметров модели и способам компенсации неоднородности верхней части разреза.

Многослойная модель предполагает последовательное определение мощности или скорости определенных интервалов разреза. Подбор объясняющих переменных для этой модели ограничен только вертикальным временем отражений. Модель идеальна для слоев с постоянной скоростью, но результат прогноза непредсказуем в случае латеральной скоростной неоднородности слоев. 
Многовариантность скоростной модели в задаче структурных построений...

В каждом конкретном случае подбор скоростной модели среды представляет нетривиальную проблему, включающую также анализ методики кинематических преобразований волнового поля в процессе обработки сейсмических данных.

\section{ЛИТЕРАТУРА}

1. Бронштейн И.Н. Справочник по математике / И.Н.Бронштейн, К.А.Семендяев. М.: Наука, 1986. 543 с.

2. Гольдин С.В. Теория интерпретации в сейсморазведке и сейсмологии. Новосибирск: ИНГГ СО РАН, 2011. 357 с.

3. Доугерти К. Введение в эконометрику: Пер. с англ. М.: ООО «Издательский дом ИНФРА-М», 1999. 401 с.

4. Мусатов И.В. Построение трехмерной скоростной модели на основе совместного анализа данных ВСП, наземной сейсморазведки и ГИС / И.В.Мусатов, А.В.Новокрещин, В.П.Торгашов // Технологии сейсморазведки. 2016. № 1. С. 5-13.

5. Система кинематической интерпретации волн (КИНГ) / Д.И.Судварг, Л.Г.Киселева, В.С.Черняк, Т.В.Курдюкова, А.Ф.Глебов. Новосибирск: Институт геологии и геофизики СО РАН, 1987. 180 с.

6. Современный подход к построению глубинно-скоростных моделей для миграции с учетом анизотропии / А.Сахаров, В.Солган, Е.Королев, Е.Вороновичева // «Геомодель-2008». Россия, г. Геленджик, 10-14 сентября, 2008 // http://0003uhk.rcomhost.com/files_public (дата обращения 10.09.2017).

7. Сысоев А.П. Прикладные задачи компенсации неоднородности верхней части разреза при обработке и интерпретации сейсмических данных. Новосибирск: ИНГГ им. А.А.Трофимука СО РАН, 2011. 90 с.

8. Jones Ian F. Anisotropic Ambiguities in TI media / Ian F.Jones, Mike L.Bridson, Nick Bernitsas // http://www.iongeo.com /content/documents/Resource\%20Center/Technical\% 20Hahers/TP_FB (дата обращения 10.09.2017).

Автор А.П.Сысоев, д-р техн. наук, профессор, sуsоеv@spmi.ru (Санкт-Петербургский горный университет, СанктПетербург, Россия).

Статья поступила в редакијию 12.09.2017

Статья принята к публикации 31.08.2018. 